Bulletin of the Section of Logic

Volume 50/3 (2021), pp. 325-335

https://doi.org/10.18778/0138-0680.2021.15

Gemma Robles* (iD)

José M. Méndez**

\title{
A NOTE ON GÖDEL-DUMMET LOGIC LC
}

\begin{abstract}
Let $A_{0}, A_{1}, \ldots, A_{n}$ be (possibly) distintict wffs, $n$ being an odd number equal to or greater than 1. Intuitionistic Propositional Logic IPC plus the axiom $\left(A_{0} \rightarrow\right.$ $\left.A_{1}\right) \vee \ldots \vee\left(A_{n-1} \rightarrow A_{n}\right) \vee\left(A_{n} \rightarrow A_{0}\right)$ is equivalent to Gödel-Dummett logic LC. However, if $n$ is an even number equal to or greater than 2, IPC plus the said axiom is a sublogic of LC.
\end{abstract}

Keywords: Intermediate logics, Gödel-Dummett logic LC.

\section{Introduction}

Propositional Intuitionistic Logic IPC can be axiomatized as follows (cf. [5] and references therein):

Axioms:

A1. $A \rightarrow(B \rightarrow A)$

A2. $[A \rightarrow(B \rightarrow C)] \rightarrow[(A \rightarrow B) \rightarrow(A \rightarrow C)]$

A3. $(A \wedge B) \rightarrow A ;(A \wedge B) \rightarrow B$

A4. $A \rightarrow[B \rightarrow(A \wedge B)]$

*http://grobv.unileon.es.

${ }^{* *}$ https://sites.google.com/site/sefusmendez.

Presented by: Norihiro Kamide

Received: December 16, 2019

Published online: July 1, 2021

(C) Copyright by Author(s), Łódź 2021

(C) Copyright for this edition by Uniwersytet Łódzki, Łódź 2021 
A5. $A \rightarrow(A \vee B) ; B \rightarrow(A \vee B)$

A6. $(A \vee B) \rightarrow[(A \rightarrow C) \rightarrow[(B \rightarrow C) \rightarrow C]]$

A7. $(A \rightarrow \neg B) \rightarrow(B \rightarrow \neg A)$

A8. $\neg A \rightarrow(A \rightarrow B)$

Rule of inference:

Modus Ponens (MP): If $A$ and $A \rightarrow B$, then $B$

The following wffs and rule (derivable in IPC) are used in the sequel:

t1. $A \rightarrow A$

t2. $(B \rightarrow C) \rightarrow[(A \rightarrow B) \rightarrow(A \rightarrow C)]$

t3. $(A \rightarrow B) \rightarrow[(B \rightarrow C) \rightarrow(A \rightarrow C)]$

Transitivity (Trans): If $A \rightarrow B$ and $B \rightarrow C$, then $A \rightarrow C$

In what follows, regardless of a particular order or association of the $n$ implicative wffs $A_{1}, \ldots, A_{n}$ connected by $\vee$ as the sole connective, in general, we simply write $A_{1} \vee A_{2} \ldots \vee A_{n}$.

By $\mathrm{IPC}_{+}$, we refer to the negationless fragment of IPC, axiomatized by A1 through A6 and MP. Well then, in [4] it is noted that Gödel-Dummett logic LC (cf. [2], [3]) can be axiomatized by adding any of the following axiom schemes to IPC:

a1. $(A \rightarrow B) \vee(B \rightarrow A)$

a2. $(A \rightarrow B) \vee[(A \rightarrow B) \rightarrow A]$

a3. $(A \rightarrow B) \vee[(A \rightarrow B) \rightarrow B]$

a4. $[A \rightarrow(B \vee C)] \rightarrow[(A \rightarrow B) \vee(A \rightarrow C)]$

a5. $[(A \wedge B) \rightarrow C] \rightarrow[(A \rightarrow C) \vee(B \rightarrow C)]$

a6. $[[(A \rightarrow B) \rightarrow B] \wedge[(B \rightarrow A) \rightarrow A]] \rightarrow(A \vee B)$

We remark that Dummett's original axiomatization of LC is the result of adding a1 to IPC (cf. [2]). We will occasionally refer to a1 as "Dummett's axiom". 
The authors of [4] add: "An even larger number of equivalents [axioms] arises by the fact that in IPC $\vdash A \vee B$ iff $\vdash(A \rightarrow B) \wedge(B \rightarrow C) \rightarrow C$ (DR), and, more generally, $\vdash D \rightarrow A \vee B$ iff $\vdash D \wedge(A \rightarrow C) \wedge(B \rightarrow C) \rightarrow C$ (EDR)" ([2], p. 1).

The aim of this note is to increase the number of equivalent axioms recorded above by showing that, for any odd number $n$ equal to or greater than 1 and (possibly) distinct wffs $A_{1}, A_{2}, \ldots, A_{n}$, addition of

$$
A_{0} \rightarrow A_{1} \vee \ldots \vee A_{n-1} \rightarrow A_{n} \vee A_{n} \rightarrow A_{0}
$$

to IPC is an axiomatization of LC.

As a by-product of the fact just stated, it also will be shown that if in the preceding wff $n$ is an even number equal to or greater than 2, addition of it to IPC results in an intermediate logic included in (but not including) LC.

To the best of our knowledge, neither of these facts is recorded in the literature.

\section{IPC plus $(A \rightarrow B) \vee[(B \rightarrow C) \vee(C \rightarrow A)]$}

Let $A_{0}, A_{1}, \ldots A_{n}, A_{n+1}, A_{n+2}$ be (possibly) distinct wffs, $n$ being an even number equal to or greater than 2 . Consider now the following wffs:

$$
\begin{aligned}
& \alpha . A_{0} \rightarrow A_{1} \vee A_{1} \rightarrow A_{2} \vee A_{2} \rightarrow A_{0} \\
& \text { B. } A_{0} \rightarrow A_{1} \vee \ldots \vee A_{n-1} \rightarrow A_{n} \vee A_{n} \rightarrow A_{0} \\
& \gamma . A_{0} \rightarrow A_{1} \vee \ldots \vee A_{n-1} \rightarrow A_{n} \vee A_{n} \rightarrow A_{n+1} \vee A_{n+1} \rightarrow A_{n+2} \vee A_{n+2} \rightarrow A_{0}
\end{aligned}
$$

We prove:

Proposition $2.1\left(\mathrm{IPC}_{+} \& \beta\right.$ proves $\left.\alpha\right)$. The wff $\alpha$ is provable in $\mathrm{IPC}_{+}$ plus $\beta$.

PROOF:

$$
\text { 1. } A_{0} \rightarrow A_{1} \vee \ldots \vee A_{n-1} \rightarrow A_{n} \vee A_{n} \rightarrow A_{0}
$$

By changing in (1), for each $i \geq 3, A_{i}$ by $A_{1}$ (resp., $A_{2}$ ) if $i$ is an odd number (resp., even number), we get

$$
\text { 2. } A_{0} \rightarrow A_{1} \vee A_{1} \rightarrow A_{2} \vee A_{2} \rightarrow A_{1} \vee A_{2} \rightarrow A_{0}
$$


or equivalently

$$
\text { 3. } A_{0} \rightarrow A_{1} \vee A_{1} \rightarrow A_{2} \vee A_{2} \rightarrow A_{0} \vee A_{2} \rightarrow A_{1}
$$

Moreover, by changing in (1), for each $i \geq 3, A_{i}$ by $A_{0}$ (resp., $\left.A_{1}\right)$ if $i$ is an odd number (resp., even number), we get

$$
\text { 4. } A_{0} \rightarrow A_{1} \vee A_{1} \rightarrow A_{2} \vee A_{2} \rightarrow A_{0} \vee A_{1} \rightarrow A_{0}
$$

Next, we proceed as follows. Obviously, we have

$$
\text { 5. }\left(A_{2} \rightarrow A_{0}\right) \rightarrow(\alpha)
$$

In addition,
6. $\left(A_{1} \rightarrow A_{0}\right) \rightarrow\left[\left(A_{2} \rightarrow A_{1}\right) \rightarrow\left(A_{2} \rightarrow A_{0}\right)\right]$
t2
7. $\left(A_{1} \rightarrow A_{0}\right) \rightarrow\left[\left(A_{2} \rightarrow A_{1}\right) \rightarrow(\alpha)\right]$
t2, Trans, 5, 6
8. $(\alpha) \rightarrow\left[\left(A_{2} \rightarrow A_{1}\right) \rightarrow(\alpha)\right]$

Then,

$$
\text { 9. }\left(A_{2} \rightarrow A_{1}\right) \rightarrow(\alpha) \quad \mathrm{A} 6,4,7,8
$$

Now, by using

$$
\text { 10. }(\alpha) \rightarrow(\alpha)
$$

3, 9 and A6, we derive

$$
\text { 11. } A_{0} \rightarrow A_{1} \vee A_{1} \rightarrow A_{2} \vee A_{2} \rightarrow A_{0}
$$

as it was to be proved.

Proposition $2.2\left(\mathrm{IPC}_{+} \& \alpha\right.$ proves $\left.\beta\right)$. The wff $\beta$ is provable in $\mathrm{IPC}_{+}$ plus $\alpha$.

Proof: Firstly, we show,

(I) The wff $\delta, A_{0} \rightarrow A_{1} \vee A_{1} \rightarrow A_{2} \vee A_{2} \rightarrow A_{3} \vee A_{3} \rightarrow A_{4} \vee A_{4} \rightarrow A_{0}$, is provable in $\mathrm{IPC}_{+}$plus $\alpha$ :

$$
\begin{aligned}
& \text { 1. } A_{0} \rightarrow A_{1} \vee A_{1} \rightarrow A_{2} \vee A_{2} \rightarrow A_{0} \\
& \text { 2. } A_{2} \rightarrow A_{3} \vee A_{3} \rightarrow A_{4} \vee A_{4} \rightarrow A_{2}
\end{aligned}
$$
$\alpha$ 
We trivially have:

$$
\begin{aligned}
& \text { 3. }\left(A_{0} \rightarrow A_{1} \vee A_{1} \rightarrow A_{2}\right) \rightarrow(\delta) \\
& \text { 4. }\left(A_{2} \rightarrow A_{3} \vee A_{3} \rightarrow A_{4}\right) \rightarrow(\delta) \\
& \text { 5. }\left(A_{4} \rightarrow A_{0}\right) \rightarrow(\delta)
\end{aligned}
$$

Then, we get

$$
\text { 6. }\left[\left(A_{4} \rightarrow A_{2}\right) \rightarrow(\delta)\right] \rightarrow(\delta) \quad \mathrm{A} 6,2,4
$$

In addition,

$$
\begin{aligned}
& \text { 7. }\left(A_{2} \rightarrow A_{0}\right) \rightarrow\left[\left(A_{4} \rightarrow A_{2}\right) \rightarrow\left(A_{4} \rightarrow A_{0}\right)\right] \\
& \text { 8. }\left(A_{2} \rightarrow A_{0}\right) \rightarrow\left[\left(A_{4} \rightarrow A_{2}\right) \rightarrow(\delta)\right] \\
& \text { 9. }\left(A_{2} \rightarrow A_{0}\right) \rightarrow(\delta)
\end{aligned}
$$

Finally,

10. $A_{0} \rightarrow A_{1} \vee A_{1} \rightarrow A_{2} \vee A_{2} \rightarrow A_{3} \vee A_{3} \rightarrow A_{4} \vee A_{4} \rightarrow A_{0} \quad \mathrm{~A} 6,1,3,9$

(II) Given (I), the wff $\varepsilon, A_{0} \rightarrow A_{1} \vee A_{1} \rightarrow A_{2} \vee A_{2} \rightarrow A_{3} \vee A_{3} \rightarrow$ $A_{4} \vee A_{4} \rightarrow A_{5} \vee A_{5} \rightarrow A_{6} \vee A_{6} \rightarrow A_{0}$, is provable in IPC + plus $\alpha$ similarly as $\delta$ has been proved above. We can use $\delta, \alpha$ and $\mathrm{t} 2$ in the forms $A_{4} \rightarrow$ $A_{5} \vee A_{5} \rightarrow A_{6} \vee A_{6} \rightarrow A_{4}$ and $\left(A_{4} \rightarrow A_{0}\right) \rightarrow\left[\left(A_{6} \rightarrow A_{4}\right) \rightarrow\left(A_{6} \rightarrow A_{0}\right)\right]$, respectively.

(III) In this way, the wff $\gamma$, displayed at the beginning of the section, can be obtained given $\beta$ (i.e., $A_{0} \rightarrow A_{1} \vee \ldots \vee A_{n-1} \rightarrow A_{n}$ ), and $\alpha$ and $\mathrm{t} 2$ in the forms $A_{n} \rightarrow A_{n+1} \vee A_{n+1} \rightarrow A_{n+2} \vee A_{n+2} \rightarrow A_{n}$ and $\left(A_{n} \rightarrow A_{0}\right) \rightarrow$ $\left[\left(A_{n+2} \rightarrow A_{n}\right) \rightarrow\left(A_{n+2} \rightarrow A_{0}\right)\right]$, respectively.

Once (I), (II) and (III) are proved, it is clear that $\beta$ is derivable from $\mathrm{IPC}_{+}$plus $\alpha$.

Given Propositions 2.1 and 2.2, we have the following corollary.

Corollary 2.3 (IPC \& $\alpha$ is equivalent to IPC \& $\beta$ ). Let $A_{0}, A_{1}, \ldots, A_{n}$ be (possibly) distinct wffs, $n$ being an even number equivalent to or greater than 2. The systems IPC plus $\alpha$ (i.e., $A_{0} \rightarrow A_{1} \vee A_{1} \rightarrow A_{2} \vee A_{2} \rightarrow A_{0}$ ) and IPC plus $\beta$ (i.e., $A_{0} \rightarrow A_{1} \vee \ldots \vee A_{n-1} \rightarrow A_{n} \vee A_{n} \rightarrow A_{0}$ ) are deductively equivalent. 
The section is ended by proving that Dummett's axiom $(A \rightarrow B) \vee(B \rightarrow$ $A)$ (a1) is not provable from IPC plus $(A \rightarrow B) \vee[(B \rightarrow C) \vee(C \rightarrow A)]$. Let us provisionally name $\mathrm{LC}_{2}$ the result of adding $(A \rightarrow B) \vee[(B \rightarrow$ $C) \vee(C \rightarrow A)]$ to IPC. We have:

Proposition 2.4 (Dummett's axiom is not provable in $\mathrm{LC}_{2}$ ). Dummett's axiom $(A \rightarrow B) \vee(B \rightarrow A)$ is not provable in $\mathrm{LC}_{2}$, that is, the result of adding $(A \rightarrow B) \vee[(B \rightarrow C) \vee(C \rightarrow A)]$ to IPC.

Proof: Consider the following set of truth-tables (4 is the only designated value):

\begin{tabular}{l|lllll|l}
$\rightarrow$ & 0 & 1 & 2 & 3 & 4 & $\neg$ \\
\hline 0 & 4 & 4 & 4 & 4 & 4 & 4 \\
1 & 2 & 4 & 2 & 4 & 4 & 2 \\
2 & 1 & 1 & 4 & 4 & 4 & 1 \\
3 & 0 & 1 & 2 & 4 & 4 & 0 \\
4 & 0 & 1 & 2 & 3 & 4 & 0
\end{tabular}

\begin{tabular}{l|lllll}
$\wedge$ & 0 & 1 & 2 & 3 & 4 \\
\hline 0 & 0 & 0 & 0 & 0 & 0 \\
1 & 0 & 1 & 0 & 1 & 1 \\
2 & 0 & 0 & 2 & 2 & 2 \\
3 & 0 & 1 & 2 & 3 & 3 \\
4 & 0 & 1 & 2 & 3 & 4
\end{tabular}

\begin{tabular}{c|ccccc}
$\vee$ & 0 & 1 & 2 & 3 & 4 \\
\hline 0 & 0 & 1 & 2 & 3 & 4 \\
1 & 1 & 1 & 3 & 3 & 4 \\
2 & 2 & 3 & 2 & 3 & 4 \\
3 & 3 & 3 & 3 & 3 & 4 \\
4 & 4 & 4 & 4 & 4 & 4
\end{tabular}

This set verifies all axioms of IPC (A1-A8) plus $(A \rightarrow B) \vee[(B \rightarrow$ $C) \vee(C \rightarrow A)]$ and the rule MP, but falsifies Dummett's axiom: let $v$ be any assignment to the propositional variables such that $v(p)=2$ and $v(q)=1$, for distinct propositional variables $p$ and $q$. Then, $v[(p \rightarrow q) \vee$ $(q \rightarrow p)]=3$.

It follows from this proposition that $\mathrm{LC}$ is not included in $\mathrm{LC}_{2}$. Instead, in the following section, it is proved that $\mathrm{LC}_{2}$ is included in $\mathrm{LC}$. 


\section{A sequence of axioms equivalent to Dummett's axiom}

Let $A_{0}, A_{1}, \ldots, A_{n}$ be distinct wffs, $n$ being an odd number equal to or greater than 1 . Now, consider the following wffs:

$$
\begin{aligned}
& \text { ह. } A_{0} \rightarrow A_{1} \vee A_{1} \rightarrow A_{0} \\
& \theta . A_{0} \rightarrow A_{1} \vee \ldots \vee A_{n-1} \rightarrow A_{n} \vee A_{n} \rightarrow A_{0}
\end{aligned}
$$

We prove:

Proposition $3.1\left(\mathrm{IPC}_{+} \& \theta\right.$ proves $\left.\varepsilon\right)$. The wff $\varepsilon$ is provable from $\mathrm{IPC}_{+}$ plus $\theta$.

Proof:

$$
\text { 1. } A_{0} \rightarrow A_{1} \vee \ldots \vee A_{n-1} \rightarrow A_{n} \vee A_{n} \rightarrow A_{0}
$$

By changing in (1), for each $i \geq 2, A_{i}$ by $A_{0}$ (resp., $A_{1}$ ) if $i$ is an even number (resp., odd number), we get

$$
\text { 2. } A_{0} \rightarrow A_{1} \vee A_{1} \rightarrow A_{0} \vee \ldots \vee A_{0} \rightarrow A_{1} \vee A_{1} \rightarrow A_{0}
$$

that is,

$$
\text { 3. } A_{0} \rightarrow A_{1} \vee A_{1} \rightarrow A_{0}
$$

i.e., the characteristic axiom of LC.

Proposition 3.2. Consider the following wff $\eta, A_{0} \rightarrow A_{1} \vee \ldots \vee A_{n-1} \rightarrow$ $A_{n} \vee A_{n} \rightarrow A_{0}$, where $A_{0}, A_{1}, \ldots, A_{n-1}, A_{n}$ are (possibly) distinct wffs. This wff $\eta$ is provable in LC (notice that $n$ is any natural number equal to or greater than 1).

ProOF:

1. $\left(A_{n} \rightarrow A_{n-1}\right) \rightarrow\left[\left(A_{n-1} \rightarrow A_{n-2}\right) \rightarrow\left(A_{n} \rightarrow A_{n-2}\right)\right]$ t3

2. $\left(A_{n} \rightarrow A_{n-2}\right) \rightarrow\left[\left(A_{n-2} \rightarrow A_{n-3}\right) \rightarrow\left(A_{n} \rightarrow A_{n-3}\right)\right]$

3. $\left(A_{n} \rightarrow A_{n-1}\right) \rightarrow\left[\left(A_{n-1} \rightarrow A_{n-2}\right) \rightarrow\left[\left(A_{n-2} \rightarrow A_{n-3}\right) \rightarrow\left(A_{n} \rightarrow A_{n-3}\right)\right]\right]$ t2, Trans, 1, 2

In this way, we have

4. $\left(A_{n} \rightarrow A_{n-1}\right) \rightarrow\left[\left(A_{n-1} \rightarrow A_{n-2}\right) \rightarrow\left[\ldots \rightarrow\left[\left(A_{1} \rightarrow A_{0}\right) \rightarrow\left(A_{n} \rightarrow A_{0}\right)\right] \ldots\right]\right]$ 
Now, we obviously have

$$
\text { 5. }\left(A_{n} \rightarrow A_{0}\right) \rightarrow(\eta)
$$

and

$$
\text { 6. }\left(A_{n-1} \rightarrow A_{n}\right) \rightarrow(\eta)
$$

So, by $\mathrm{t} 2, \mathrm{t} 3,(4)$ and (5), we derive

7. $\left(A_{n} \rightarrow A_{n-1}\right) \rightarrow\left[\left(A_{n-1} \rightarrow A_{n-2}\right) \rightarrow\left[\ldots \rightarrow\left[\left(A_{1} \rightarrow A_{0}\right) \rightarrow(\eta)\right] \ldots\right]\right]$

And by A1, (6) and Trans, we obtain

8. $\left(A_{n-1} \rightarrow A_{n}\right) \rightarrow\left[\left(A_{n-1} \rightarrow A_{n-2}\right) \rightarrow\left[\ldots \rightarrow\left[\left(A_{1} \rightarrow A_{0}\right) \rightarrow(\eta)\right] \ldots\right]\right]$

Now, by Dummett's axiom, we have

$$
\text { 9. }\left(A_{n-1} \rightarrow A_{n}\right) \vee\left(A_{n} \rightarrow A_{n-1}\right)
$$

whence

$$
\text { 10. }\left(A_{n-1} \rightarrow A_{n-2}\right) \rightarrow\left[\left(A_{n-2} \rightarrow A_{n-3}\right) \rightarrow\left[\ldots \rightarrow\left[\left(A_{1} \rightarrow A_{0}\right) \rightarrow(\eta)\right] \ldots\right]\right]
$$

follows by A6, (7), (8) and (9).

Next, notice that, for any $k(0 \leq k \leq n-1)$,

$$
\text { 11. }\left(A_{k} \rightarrow A_{k+1}\right) \rightarrow(\eta)
$$

is clearly provable.

Finally, proceeding from (10) and (11), similarly as we have proceeded from (4), (7), (8) and (9) to (10), we eventually derive

$$
\text { 12. } A_{0} \rightarrow A_{1} \vee \ldots A_{n-1} \rightarrow A_{n} \vee A_{n} \rightarrow A_{0}
$$

that is, the wff $\eta$, as it was to be proved.

Given Propositions 3.1 and 3.2, we immediately have the following corollary.

Corollary 3.3 (IPC \& $\theta$ is equivalent to LC). Let $A_{0}, A_{1}, \ldots, A_{n}$ be (possibly) distinct wffs, $n$ being an odd number equivalent to or greater than 1. The result of adding the wff $\theta$ (i.e., $A_{0} \rightarrow A_{1} \vee \ldots A_{n-1} \rightarrow A_{n} \vee A_{n} \rightarrow$ $\left.A_{0}\right)$ to IPC is a system deductively equivalent to LC. 
On the other hand, given Propositions 2.4 and 3.2, the following corollary is immediate.

Corollary $3.4\left(\mathrm{LC}_{2}\right.$ is included in $\left.\mathrm{LC}\right)$. The system $\mathrm{LC}_{2}$, that is, IPC plus the axiom $(A \rightarrow B) \vee[(B \rightarrow C) \vee(C \rightarrow A)]$ is included in (but does not include) LC.

\section{A couple of remarks}

This note is ended with a couple of remarks.

1. The proofs of Propositions 2.1, 2.2, 3.1 and 3.2 are given within the context of $\mathrm{IPC}_{+}$, but it is possible that weaker systems are sufficient. For example, MaGIC (cf. [7]) does not find a set of truth-tables verifying Ticket Entailment (cf. [1]) plus Dummett's axiom but falsifying $(A \rightarrow B) \vee(B \rightarrow$ $C) \vee(C \rightarrow D) \vee(D \rightarrow A)$.

2. An IPC-model is the following structure $(K, R, \models)$, where $K$ is a nonempty set, $R$ is a reflexive and transitive binary relation defined on $K$ and $\vDash$ is a (valuation) relation such that for each $a \in K$, propositional variable $p$ and wffs $A, B$, the following conditions (clauses) are fulfilled:

(i) $(R a b \& a \vDash p) \Rightarrow b \vDash p$

(ii) $a \vDash A \wedge B$ iff $a \vDash A$ and $a \vDash B$

(iii) $a \vDash A \vee B$ iff $a \vDash A$ or $a \vDash B$

(iv) $a \vDash A \rightarrow B$ iff for all $b \in K,(\operatorname{Rab}$ and $b \vDash A) \Rightarrow b \vDash B$

(v) $a \vDash \neg A$ iff for all $b \in K, R a b \Rightarrow b \not \models A$

We have: for any set of wffs $\Gamma$ and wff $A, \Gamma \vdash_{\text {IPC }} A$ iff $\Gamma \vDash A(\Gamma \vDash A$ iff for any IPC-model $\mathcal{M}$ and $a \in K, a \vDash A$ if $a \vDash \Gamma$, where $a \vDash \Gamma$ iff $a \vDash B$ for all $B \in \Gamma$ ) (cf. [5] or [6] and references therein).

Well then, let us name $\mathrm{LC}_{n}$ the result of adding the axiom

$$
A_{0} \rightarrow A_{1} \vee \ldots \vee A_{n-1} \rightarrow A_{n} \vee A_{n} \rightarrow A_{0}
$$

to IPC; and let $\mathrm{LC}_{n}$-models be the result of adding the following condition to IPC-models: for any $a_{0}, a_{1}, \ldots, a_{n} \in K$, if $R a_{0} a_{1}$ and $R a_{0} a_{2}$ and,$\ldots$, and $R a_{0} a_{n}$, then, $R a_{1} a_{n}$ or $R a_{2} a_{1}$ or , ..., or $R a_{n} a_{n-1}$. For instance, $\mathrm{LC}_{2^{-}}$ models (i.e., models for IPC plus the axiom $(A \rightarrow B) \vee(B \rightarrow C) \vee(C \rightarrow A))$ 
are defined when adding to IPC-models the condition, for any $a, b, c, d \in K$, $(R a b \& R a c \& R a d) \Rightarrow(R b d$ or $R c b$ or $R d c)$. It is not difficult to prove that $\mathrm{LC}_{n}$ is (strongly) sound and complete w.r.t. $\mathrm{LC}_{n}$-models.

Acknowledgements. Work supported by research project FFI201782878-P financed by the Spanish Ministry of Economy, Industry and Competitiveness. We sincerely thank two anonymous referees of the Bulletin of the Section of Logic for their comments and suggestions on a previous draft of this paper.

\section{References}

[1] A. R. Anderson, N. D. Belnap Jr., Entailment. The Logic of Relevance and Necessity, vol. I, Princeton University Press, Princeton, NJ (1975).

[2] M. Dummett, A propositional calculus with denumerable matrix, Journal of Symbolic Logic, vol. 24(2) (1959), pp. 97-106, DOI: https://doi.org/10. $2307 / 2964753$.

[3] K. Gödel, Zum intuitionistischen Aussagenkalkül, Anzeiger der Akademie der Wissenschaften in Wien, vol. 69 (1932), pp. 65-66.

[4] D. D. Jongh, F. S. Maleki, Below Gödel-Dummett, [in:] Booklet of abstracts of Syntax meets Semantics 2019 (SYSMICS 2019), Institute of Logic, Language and Computation, University of Amsterdam (2019), pp. 99-102.

[5] J. Moschovakis, Intuitionistic Logic, [in:] E. N. Zalta (ed.), The Stanford Encyclopedia of Philosophy, winter 2018 ed. (2018), URL: https://plato. stanford.edu/archives/win2018/entries/logic-intuitionistic.

[6] G. Robles, J. M. Méndez, A binary Routley semantics for intuitionistic De Morgan minimal logic HM and its extensions, Logic Journal of the IGPL, vol. 23(2) (2014), pp. 174-193, DOI: https://doi.org/10.1093/jigpal/jzu029.

[7] J. K. Slaney, MaGIC, Matrix Generator for Implication Connectives: Version 2.1, Notes and Guide (1995), http://users.cecs.anu.edu.au/jks/magic.html. 


\section{Gemma Robles}

University of León

Department of Psychology, Sociology and Philosophy

Campus de Vegazana, s/n, 24071

León, Spain

e-mail: gemma.robles@unileon.es

José M. Méndez

University of Salamanca

Campus Unamuno

Edificio FES, 37007

Salamanca, Spain

e-mail: sefus@usal.es 